\title{
ÉVOLUTION PARALLÈLE ET CONVERGENCE : LE LANGAGE ESTHÉTIQUE DE L'ABSTRACTION DANS LES \\ AVEUGLES DE M. MAETERLINCK ET DANS DU SPIRITUEL DANS L'ART DE W. KANDINSKY
}

Nathalie Arcand, Université McGill

\section{Résumé analytique}

En concevant sa théorie de l'abstraction, élaborée pour la première fois dans son ouvrage Du Spirituel dans l'art publié en 1911, le peintre Wassily Kandinsky exprime une profonde admiration pour le premier théâtre de Maurice Maeterlinck. Cette dernière découle d'une réflexion sur le langage esthétique, désormais soumis à un processus complexe d'épuration : le mot chez Maeterlinck, tout comme les éléments géométriques et les couleurs en peinture, affirme son autonomie par rapport à la réalité extérieure et empirique à laquelle il se référait antérieurement. Cette transformation de la fonction du signe artistique, lexical et pictural, sera illustrée au moyen du drame Les Aveugles.

Souvent, les peintres préconisant l'abstraction dans l'art se sont penchés sur ce que leurs théories devaient aux générations précédentes. Wassily Kandinsky, un des fondateurs de l'art abstrait, fait le pont entre deux générations : ses années de formation sont imprégnées d’une esthétique symboliste, alors que lui-même n'atteint la maturité artistique qu'au début des années 1910. À cette époque, le peintre est à la recherche d'un nouveau langage esthétique, axé sur le rejet de toute référence à la réalité empirique. Kandinsky perçoit le dramaturge symboliste, Maurice Maeterlinck, en tant qu'un des fondateurs de ce nouveau langage. Tel qu'il le démontre dans Du Spirituel dans l'art, et dans la peinture en particulier, Kandinsky est sensible au rejet de la matérialité dans les œuvres théâtrales de Maeterlinck et remarque une autonomie naissante entre le signe lexical et l'objet qu'il désigne. C'est donc en littérature, au niveau du langage, que Kandinsky repère les premiers indices d'un art purement abstrait: comme il l'exprime lui-même, «la grande ressource de Maeterlinck est le mot» (Kandinsky Du Spirituel 81) ${ }^{1}$. Le signe linguistique se délivre peu à peu de sa fonction référentielle et se libère ainsi de la gangue du matérialisme. D'une part, le mot comporte un sens direct, utilitaire, concret, brut, servant à simplement dénommer l'objet; d'autre part, il possède un sens abstrait, intérieur, parlant à l'âme. Cette ambivalence entre une réalité «extérieure », matérielle, et une sphère « intérieure », spirituelle, illustre en même temps deux facettes de l'expérience humaine : Kandinsky fait de cette ambivalence le fondement de sa théorie de l'art abstrait. Son objectif est de rompre la connexion entre I'élément pictural, I'œil et le visible : de cette façon, il cherche à renoncer à la tradition de l'art figuratif selon laquelle le peintre doit reproduire ce qui apparaît au dehors, une conception de l'art transmise par la Grèce antique, que le critique Michel Henry résume ainsi :

I'Idée grecque qui se tient à la source de ce réseau de relations et d'implications, c'est celle du phénomène justement. 'Phénomène' pour les Grecs désigne ce qui brille, ce qui se montre dans la lumière $[. .$.$] . Ce qui montre, ce qui fait voir, c'est donc la lumière elle-même. Voir, c'est avoir part à la$ lumière, c'est entrer en elle, être éclairé par elle - c'est être dans le monde. (Henry 19)

Cependant, selon Maeterlinck et Kandinsky, si le mot, tout comme les éléments picturaux, est destiné à fournir une représentation du visible, il se retrouve subordonné à un modèle préexistant et, par là, réduit à servir de réplique. Selon la théorie de Kandinsky, tout comme dans le cas de l'autonomie du signe linguistique observée chez Maeterlinck, le concept de «phénomène » est désormais à l'abri des injonctions imposées par le visible et mis en relation avec la dimension spirituelle et donc « invisible » de la vie. Le « principe invisible » (Maeterlinck 
«Le réveil de l'âme » 29) que décrit Maeterlinck dans son essai «Le réveil de l'âme » est équivalent à I' «Essentiel Intérieur» (Kandinsky Du Spirituel 52) pour Kandinsky: mais cette dimension de l'existence humaine ne peut être atteinte et exprimée qu'après un long processus servant à débarrasser l'œuvre d'art de toute référence au monde extérieur. L'abstraction comporte ainsi deux étapes principales: d'abord, les «moyens » artistiques, notamment le mot, la forme et la couleur, sont extraits et isolés de leurs fonctions mimétiques dans le langage traditionnel; puis, l'étape de la purification de ces moyens permet un retour vers des éléments primordiaux, occasionnant ultimement une renaissance du langage esthétique. Le drame Les Aveugles de Maeterlinck servira de modèle afin d'illustrer ce processus

D’emblée, Maeterlinck et Kandinsky rejettent la dimension traditionnellement anthropocentrique de l'œuvre d'art: contrairement à ce qui est dicté par l'esthétique naturaliste, I'homme est coupé de son environnement, tandis que son comportement ne s'explique plus par la connaissance des faits. La réalité entourant les aveugles dans le drame de Maeterlinck se dérobe et se «dématérialise », modifiant profondément les capacités d'expression de I'homme. Comme le propose le critique Paul Gorceix, la scission par rapport au monde extérieur entraîne « le dénuement de la condition humaine, condamnée à errer perpétuellement dans les ténèbres de l'ignorance de son destin » (Gorceix 336-337); c'est ce qu'exprime ainsi le plus vieil aveugle : « Nous n'avons jamais vu la maison où nous vivons ; nous avons beau tâter les murs et les fenêtres ; nous ne savons pas où nous vivons!» (Maeterlinck Aveugles 58). Les personnages semblent en effet sans identité et leur entourage ne fournit aucune précision. Maeterlinck envisage, dans les «Menus Propos » datant de 1890, année de publication des Aveugles, le rejet de la dimension anthropocentrique de façon tout aussi radicale que certains peintres abstraits : au théâtre, «l'absence de l'homme me semble indispensable ». Il souligne qu' « il faudrait peut-être écarter entièrement l'être vivant de la scène » et propose de remplacer l'être humain par « une ombre, un reflet, une projection de formes symboliques d'un être qui aurait les allures de la vie sans avoir la vie » (Maeterlinck «Menus propos » 462). Le personnage maeterlinckien est réduit à l'état d'un objet, d'un fantoche ou d'un «pupazz[o] mystiqu[e]» (Artaud 96). Ces personnages semblent « sans vie », mais cela uniquement « extérieurement », car « intérieurement », une dimension invisible de l'existence se développe. À cet égard, la cécité devient une importante métaphore de l'abstraction aux yeux de nombreux artistes et penseurs au tournant du siècle, tel que le poète allemand Novalis, dont la poésie a été traduite par Maeterlinck : «Rentrer en soi, [...] s'abstraire du monde extérieur » (Novalis 140). L'abstraction devient donc synonyme d'introspection. Pour Kandinsky, selon Henry,

I'Être n'est [...] pas une notion univoque. Deux dimensions le traversent et viennent déchirer son unité primitive [...] : celle du visible où dans la lumière du monde les choses se donnent à nous et sont vécues par nous comme des phénomènes extérieurs; celle de l'invisible où, en l'absence de ce monde et de sa lumière, avant même que surgisse cet horizon d'extériorité qui met toute chose à distance de nous-même et nous la pro-pose à titre d'ob-jet (ob-jet veut dire : ce qui est posé devant), la vie s'est déjà emparée de son être propre, s'étreignant elle-même dans cette épreuve intérieure et immédiate de soi qui est son pathos, qui fait d'elle la vie. (Henry 18-19)

Les personnages sont « déshumanisés» afin de révéler «la forme et le désir de [leur] œil intérieur» (Maeterlinck «Vie profonde »143). Un phénomène ne se révèle plus uniquement « extérieurement », dans la lumière du jour, tel que le constate le premier aveugle-né : «il ne faut pas de lumière à ceux qui ne voient 
pas » (Maeterlinck Aveugles 58). Ces «deux façons » (Kandinsky Point et ligne15) décrites par Kandinsky correspondent à deux modes d'apparaître.

Kandinsky et Maeterlinck privilégient la perception intérieure, tel que démontré dans Les Aveugles, lorsque le spectateur est témoin de certains brefs moments d'accès aux « demeures intérieures de l'âme » (Gorceix 344) : «Moi, je ne vois que quand je rêve» (Maeterlinck Aveugles 59), dit le Plus Vieil Aveugle, alors que les mots de la Jeune Aveugle font penser à une forme de vision intérieure, dépourvue d'images : « Mes paupières sont fermées, mais je sens que mes yeux sont en vie... » (56). Et pourtant, dans Les Aveugles, la cécité $n$ 'interdit pas toute référence au réel; la dimension visuelle de la perception est supprimée, mais le personnage peut tout de même avoir conscience des formes, corps et bruits issus du monde naturel. Comme le remarque lui-même Kandinsky, «l'abstraction pure, comme le réalisme pur, [dont celui du Douanier Rousseau,] se sert des choses dans leur existence matérielle » (Kandinsky «Question de la forme » 157) et font face au même défi, celui de rendre visible et donc d'extérioriser, grâce à des «formes » ou «moyens artistiques », une réalité intérieure et invisible. Afin de résoudre ce paradoxe, la critique tend à accepter l'existence d'un schisme séparant la forme et le contenu, voire la suprématie du contenu sur la forme. Cependant, en abolissant cette dissociation, Kandinsky met fin au «problème de la forme »: puisque les moyens permettent de représenter le contenu invisible, ils « doivent être compris maintenant comme 'intérieurs' dans leur signification et finalement dans leur réalité véritable - comme 'invisibles' » (Henry 23). Ces deux entités sont désormais homogènes et soumises à un principe d'équivalence : la forme devient identique au contenu. Ce même principe s'applique tout autant à l'art abstrait qu'au « grand réalisme » (Kandinsky «Question de la forme» 153) du Douanier, dont Kandinsky admirait les œuvres. Alors que I'abstraction souhaite exclure la réalité objective, le « grand réalisme » se fonde sur cette dernière, mais sans la soumettre aux lois esthétiques et artificielles, étrangères à l'essence de l'art : « le grand réalisme, [...] s'efforce d'éliminer du tableau l'élément esthétique extérieur, afin d'exprimer le contenu de l'œuvre par la restitution simple ('inesthétique') de l'objet dans sa simplicité et sa nudité » (153). Les formes «abstraites » et « objectives » partagent un même objectif, celui de faire apparaître l'art dans sa pureté : après tout, comme le soutient le peintre, «ces deux pôles sont équivalents en dernière analyse. [...] La plus grande dissemblance extérieure devient la plus grande ressemblance intérieure » (155).

Si le modèle de Kandinsky ne nie pas la présence de formes « objectives » dans le domaine de l'art « pur», chez Maeterlinck, le traitement de la nature dans Les Aveugles ne mitige pas l'impact de la dimension «abstraite» de l'œuvre : après tout, le paysage ne sert aucunement de reflet ou de «concrétisation» allégorique de l'état d'âme du personnage. Les objectifs de Maeterlinck et de Kandinsky s'apparentent : I'art n'est plus un miroir proposant une image de l'être humain et de son environnement, mais, au lieu, fait coïncider le phénomène se révélant avec la perception interne du personnage; il ne s'agit plus d'une accumulation d'images servant à illustrer, grâce à un processus d'extériorisation, l'expérience humaine, mais d'un moyen d'inciter le personnage, comme le spectateur, à pénétrer le tableau lui-même et, tel que l'exprime Kandinsky dans Regards sur le passé, à « [se] mouvoir au sein même du tableau, à vivre dans le tableau » (Kandinsky 
Regards sur le passé 108). La frontière entre la nature se manifestant au devant et l'observateur devient poreuse, d'où la difficulté qu'éprouvent les aveugles à appréhender et à distinguer les phénomènes naturels, dont le tonnerre, le bruit des vagues et le bruissement du vent dans les feuilles mortes ${ }^{2}$. Ces phénomènes ne se révèlent pas à la lumière du jour; ils sont extraits de leur contexte d'origine, dissociés de leur source, de sorte qu'ils demeurent ambigus, insaisissables et indéchiffrables, tout comme en peinture, où «on voit que c'est justement d'être détachés de l'univers, du contexte objectif d'un paysage par exemple, que couleurs et graphismes tirent leur puissance envoûtante. Et cette puissance, c'est précisément leur subjectivité » (Henry 231). C'est justement la fonction de ces bruissements, des odeurs, des objets effleurés dans Les Aveugles : ces éléments extérieurs s'éprouvent intérieurement dans «le pathos de [la] subjectivité invisible» (230) se révélant progressivement dans l'âme des aveugles. L'Intérieur ne se matérialise jamais, il ne se montre pas et ne se laisse donc pas apercevoir à la manière d'un «monde ». Cette absence d'extériorité s'exprime clairement par un des aveugles lorsqu'il constate qu' «il n'y a rien à voir au-dehors! » (Maeterlinck Aveugles 45). Un nouveau mode d'expression se développe afin de pouvoir tenir compte d'un phénomène qui ne se manifeste plus devant le personnage, tel un objet «pro-posé », imposant une distance entre ce dernier et le locuteur; au lieu, l’ « œil intérieur » permet la suppression de cette distance et fait coïncider le phénomène avec la subjectivité du locuteur.

Ce rejet radical du langage traditionnel, servant à décrire la réalité empirique, donne lieu à une épuration «systématique » des signes linguistique et pictural jusqu'à leurs formes élémentaires et essentielles. En premier lieu, afin de distancier progressivement le signe de sa fonction mimétique, Maeterlinck emploie le mot pour évoquer une image rudimentaire, primitive, qui s'éloigne du «monde ». À titre d'exemple, la brève description de l'île sur laquelle se trouvent les aveugles demeure floue, énigmatique, à peine qualifiée, fournissant une image davantage fantastique et surréelle des environs et évoquant un espace primordial. Selon la didascalie, le drame a lieu dans une «très ancienne forêt septentrionale, d'aspect éternel sous un ciel profondément étoilé » (Maeterlinck Aveugles 35) :

il [le prêtre] disait qu'il nous fallait connaître un peu la petite île où nous sommes. Lui-même ne l'a jamais entièrement parcourue; il y a une montagne où personne n'a monté, des vallées où l'on n'aime pas à descendre et des grottes où nul n'a pénétré jusqu'ici. Il disait enfin qu'il ne fallait pas toujours attendre le soleil sous les voûtes du dortoir; il voulait nous mener jusqu'au bord de la mer. (45)

La nature est ainsi évoquée, mais comme le dit Gorceix, les divers éléments ont été choisis « en fonction de leur effet sur l'imaginaire du spectateur. [...] Maeterlinck a pris soin d'ôter ce que la nature pouvait avoir de réel pour en faire une sorte de symbole de l'infini, à la limite du fantastique» (Gorceix 338). En deuxième lieu, comme le remarque Kandinsky dans Du Spirituel dans l'art, Maeterlinck omet de la scène et du décor un objet évoqué par un mot :

quand certains de ses drames furent montés à Saint-Pétersbourg sous sa propre direction, Maeterlinck utilisa, au cours d'une répétition, un simple morceau de toile pour remplacer un élément de décor une tour - manquant. Il ne lui semblait pas nécessaire de faire fabriquer un décor avec tous les détails. [...] Cette tendance à stimuler l'imagination du spectateur joue un grand rôle dans le théâtre contemporain. [...] C'est là une transition nécessaire du matériel au spirituel dans le théâtre de I'avenir. (Kandinsky Du Spirituel 81) 
Dans les deux cas, l'objet évoqué par le mot correspond à une entité qui n'est pas imitative, se faisant appréhender et interpréter selon la subjectivité du personnage. Ces deux «techniques » contribuent à la mise en scène d'un univers « indéfinissable » selon les critères extérieurs, rendant ainsi tout aussi abstraite et donc « intérieure » que possible la réalité évoquée par le mot, une figuration reléguée à l'espace imaginaire, voire fantastique, l'emportant sur la figuration « réaliste ».

Pourtant, ces exemples d'un processus d'épuration ne concernent que la réalité évoquée grâce au mot, et non le signe linguistique, I'outil lui-même. Ainsi, malgré l'omniprésence de la cécité et la dissociation progressive des images suscitées du «monde», la dimension « extérieure » n'est toujours pas entièrement omise, c'est-à-dire, comme l'exprime Henry, «le monde tel qu'il surgit dans sa visibilité incontestable, qui ne cesse de m'offrir son spectacle et auquel, même lorsque je ferme les yeux, j'adhère encore par tous mes sens levés vers lui »(Henry 17). Aussi Maeterlinck franchit-il encore une autre limite dans le processus d'épuration du signe linguistique pour en arriver à l'étape précédant même l'acte d'énonciation: le silence. L'acte d'énonciation permet désormais de divulguer une strate superficielle de sens, alors que le silence, le non-dit, devient le détenteur des vérités mystiques et profondes : il s'agit de la dimension la plus radicalement nonfigurative de son théâtre. Désormais, comme l'exprime le critique Michel Viegnes, «[I]e but suprême de la parole est donc d'être une antichambre du silence, que Maeterlinck désigne comme 'l'ange des vérités suprêmes' » (Viegnes 76) : selon le dramaturge, «le silence est l'élément dans lequel se forment les grandes choses, pour qu'enfin elles puissent émerger, parfaites et majestueuses, à la lumière de la vie qu'elles vont dominer » (Maeterlinck « Silence » 15). Le silence, nouveau signe linguistique, devient un « élément de base », termes employés par Kandinsky dans Point et ligne sur plan, « c'est-à-dire ceux sans lesquels aucune œuvre ne peut naître dans tel ou tel domaine de l'art » (Kandinsky Point et ligne 20). Pour sa part, Kandinsky passe à la réduction des éléments picturaux à des couleurs et à des formes géométriques pures. Si le silence constitue I'élément de base pour Maeterlinck, Kandinsky perçoit le point en tant qu' « élément originel de la peinture » (21), ce dernier pouvant se transformer en surface ou en ligne. D'après le peintre, le point a comme équivalant le silence : « le point géométrique est un être invisible. Il doit donc être défini comme immatériel. Du point de vue matériel le point égale Zéro. [...] Selon notre conception, ce Zéro - le point géométrique - évoque la concision absolue, c'est-à-dire la plus grande retenue, mais qui parle cependant » (25). Tout comme dans le théâtre de Maeterlinck, il s'agit d'un silence «parlant», révélateur: Kandinsky soutient que «le point géométrique est [...] l'ultime et unique union du silence et de la parole. C'est pour cela que le point géométrique a trouvé sa forme matérielle en premier lieu dans l'écriture - il appartient au langage et signifie silence »(25). C'est ainsi que le dialogue des aveugles est fondé sur un échange de propos n'ayant plus de fonction dynamique et communicative : il acquiert avant tout une valeur suggestive, servant à évoquer I'indéfinissable et l'irreprésentable. Le dialogue des Aveugles comporte une grande quantité de sous-entendus, de répétitions de phonèmes, d'espaces, de vides et de points de suspension. C'est au lecteur-spectateur de compenser ces «blancs», une activité qui sert de charnière entre le silence et la parole: le silence chez 
Maeterlinck, tout comme le point pour Kandinsky, n'est pas l'ultime produit d'un processus de déconstruction, mais un commencement.

Une telle épuration radicale du signe linguistique pourrait mener à un appauvrissement de la langue, d'où l'angoisse profonde des personnages maeterlinckiens face à ce silence envahissant qui leur enlève temporairement toute capacité d'énonciation à travers le langage traditionnel : «J'ai peur quand je ne parle pas » (Maeterlinck Aveugles 39), s'exclame un des aveugles. Devant une composition kandinskienne abstraite, le spectateur éprouve ce même genre de désolation en observant la réduction radicale des formes picturales et des couleurs à des «éléments de base ». Si, dans Les Aveugles, I'univers entourant le locuteur demeure inexprimable, chez Kandinsky, le spectateur est confronté à un univers méconnaissable : le peintre décrit cette réaction ainsi dans son essai «Sur la question de la forme »: « d'une part, l'artiste ôte à l'élément abstrait I'appui anecdotique qu'il prend sur l'élément objectif et laisse le public dans l'incertitude. On dit: l'art abandonne la terre ferme. D'autre part, l'artiste écarte, par l'abstraction, toute idéalisation anecdotique de l'élément objectif de sorte que le public se sent rivé au sol. On dit : I'art abandonne l'idéal » (Kandinsky «Question de la forme » 153). Et pourtant, ce processus d'abstraction ne doit pas mener à une affirmation du néant, ni à la limitation de l'existence humaine. Pour cette raison, comme nous le rappelle le peintre dans Point et ligne sur plan, «l'opinion, répandue aujourd'hui encore, qu'il serait fatal de 'disséquer' l'art, et que cette autopsie mènerait inévitablement à la mort de l'art, résulte de l'ignorante dépréciation des éléments mis à nu et de leurs forces primaires » (Kandinsky Point et ligne 16). Cette analyse essentielle ne signifie pas pour autant « décomposition», mais plutôt la «vie», car elle «nous reconduit à l'essence de la chose, en l'occurrence l'élément pictural pur. Or l'essence de l'élément pictural est justement le contenu abstrait, vie invisible que cet élément veut exprimer» (Henry 61). Ainsi, l'épuration occasionne une renaissance du langage à partir d'éléments primordiaux, dont le silence et le point, devenus matière originelle, délivrée des contraintes superficielles du monde extérieur.

Toujours est-il que si cette pensée libératrice, axée sur un retour aux essences, sous-tend tout l'art kandinskien, en revanche, le premier théâtre de Maeterlinck, dont Les Aveugles nous fournit un excellent exemple, illustre un monde étouffant, emprisonnant et angoissant. Cette contradiction n'est pourtant qu'apparente : le sentiment de disjonction et de précarité représenté chez Maeterlinck ainsi que la notion de libération véhiculée par l'œuvre de Kandinsky font partie d'un même processus, équivalant à la conception kandinskienne de l'expérience de l'art abstrait. Le peintre propose la description suivante de la dimension répressive du théâtre de Maeterlinck :

notre âme, après la longue période de matérialisme dont elle ne fait que s'éveiller, recèle les germes du désespoir, de l'incrédulité, de l'absurde et de l'inutile. Le cauchemar des doctrines matérialistes, qui a fait de la vie de l'univers un jeu stupide et vain, n'est pas encore dissipé. Revenant à soi, l'âme reste oppressée. Seule une faible lumière vacille comme un point minuscule dans un énorme cercle du Noir. Cette faible lumière n'est qu'un pressentiment et l'âme n'a pas le courage de la voir dans le doute que cette lumière soit le rêve, et le cercle du Noir. (Kandinsky Du Spirituel 52)

Ce processus de rupture évoque une série de caractéristiques du premier théâtre de Maeterlinck, proposée par le critique Delphine Cantoni, cadrant tout à fait avec la description d'une œuvre abstraite : « ce premier théâtre [...] est donc placé sous le signe de la disjonction, de la précarité, de la séparation; il est saturé à tous les 
niveaux (textuels, scéniques, iconiques) par le vide, la distance, l'épuration » (Cantoni 20). Et pourtant, au cœur même du concept kandinskien de l'abstraction et du rejet de l'anthropocentrisme par Maeterlinck se fait voir une transition du « segment », de l' « interstice », au « liant », selon la formule proposée par Cantoni :

en dépit de cette insistance sur l'intervalle et la distance s'impose le sentiment d'une continuité organique confuse entre les personnages, voire entre ceux-ci et les lecteurs ou spectateurs, une sorte de lien vivant, [...] et qui assume à lui seul les modalités de la communication, frappée d'ostracisme dans les domaines dont elle a d'ordinaire l'apanage tels que le langage, le statut psychologique du personnage ou l'occupation de l'espace par les corps [...]. (20)

Aussi, selon cette perspective, les œuvres du dramaturge et du peintre se complémentent-elles en illustrant la nature double du phénomène de l'abstraction.

Ce sont les « éléments de base » du langage, soit le silence ou le point qui assurent le développement de ces nouvelles modalités de la communication et, en même temps, la transition d'un état à l'autre. C'est ce qu'exprime Maeterlinck dans son essai «Le Silence » lorsqu'il décrit le passage du silence passif au silence actif :

Je ne m'approche ici que du silence actif, car il y a un silence passif, qui n'est que le reflet du sommeil, de la mort ou de l'inexistence. C'est le silence qui dort; et tandis qu'il sommeille, il est moins redoutable encore que la parole; mais une circonstance inattendue peut l'éveiller soudain, et alors c'est son frère, le grand silence actif, qui s'intronise. [...] Et c'est parce qu'aucun de nous n'ignore cette sombre puissance et ses jeux dangereux que nous avons une peur si profonde du silence. Nous supportons à la rigueur le silence isolé, notre propre silence : mais le silence de plusieurs, le silence multiplié, et surtout le silence d'une foule est un fardeau surnaturel dont les âmes les plus fortes redoutent le poids inexplicable. (Maeterlinck «Silence »17)

Pour cette raison, les aveugles préfèrent un espace clos, le «silence isolé »: «j'aime mieux ne pas sortir » (Maeterlinck Aveugles 49), dit l'un d'entre eux. Mais grâce au silence actif qui s'impose, l'éveil de la dimension à la fois infinie et unificatrice de l'existence humaine, le «liant», demeure latent: «je soupçonne alors de grandes clartés; et mes yeux font de grands efforts pour s'ouvrir » (50), ou encore, «je croyais que j'allais y voir tout à coup »(75), disent les personnages. Le point peut aussi être, dans le langage, le symbole de I'interruption ainsi que le «pont d'un Être à l'autre » (Kandinsky Point et ligne 25) :

en isolant petit à petit le point du cercle restreint de son action habituelle [c'est-à-dire de sa fonction liée à l'écriture], ses caractéristiques intérieures, muettes jusqu'alors, dégagent une résonance accrue. Ses caractéristiques - tensions intérieures - se dégagent une à une des profondeurs de son être et leurs forces rayonnent. Leurs effets et influences surmontent les refoulements humains. Bref - le point mort devient un être vivant. (28)

Ainsi, de la même façon, les objets quotidiens auparavant inertes parsemant le drame de Maeterlinck émettent une certaine résonance, se laissant capter grâce à cette «fluidité organique » qui permet la communion d'une âme à l'autre et qui «fusionne les vivants en la plénitude d'une vie unique et close» (Cantoni 23). Certains personnages s'expriment ainsi : «je crois qu'il y a des étoiles ; je les entends » (Maeterlinck Aveugles 47); «il me semble que je sens le clair de lune sur mes mains » (46); la plus vieille aveugle dit à l'égard de l'aveugle folle, «je l'entends vivre »(70). Le passage de la disjonction à la symbiose rejoint les propos de Kandinsky concernant l'ambition de toute recherche, de toute quête d'apprentissage, fondée, en un premier temps, sur « I'examen minutieux de chaque phénomène » (Kandinsky Point et ligne 21), puis sur une synthèse de ces phénomènes isolés. L'œuvre de Maeterlinck exemplifie ce même processus, la rupture initiale d'avec la tradition de l'art mimétique servant de pont vers la vie intérieure de l'œuvre et de l'existence humaine. La vision esthétique de ces deux artistes permet de réfuter les rapports antithétiques que l'on établit souvent entre l'art 
abstrait et l'être humain: au lieu, parce que l'homme se voit muni de nouvelles capacités d'expression, Maeterlinck peut être perçu en tant que précurseur du concept de l'« humanité » de l'art abstrait à ses origines.

\section{Bibliographie}

Artaud, Antonin. « Maurice Maeterlinck. » La Fenêtre ardente (2) (1974) : 96-97.

Breton, André. « Genèse et perspective artistiques du surréalisme. » Le Surréalisme et la peinture. Paris : Gallimard, 1965. 49-82.

Cantoni, Delphine. «Le premier théâtre de Maeterlinck : du segment au liant. » Nord'(26) (décembre 1995) : 19-33.

Gorceix, Paul. Maeterlinck : I'arpenteur de l'invisible. Bruxelles : Le Cri édition, 2005.

Henry, Michel. Voir l'invisible sur Kandinsky. Paris : PUF, 2005.

Kandinsky, Wassily. Du Spirituel dans l'art, et dans la peinture en particulier. Philippe Sers, trad. Paris : Éditions Denoël, 1989.

Kandinsky, Wassily. Point et ligne sur plan, Suzanne et Jean Leppien, trad. Paris : Éditions Gallimard, 1991.

Kandinsky, Wassily. «Sur la question de la forme. » Regards sur le passé et autres textes 1912-1922. Jean-Paul Bouillon, éd. Paris : Hermann, 2003. 145-167.

Maeterlinck, Maurice. «Le réveil de l'âme. » Le Trésor des humbles. Bruxelles : Éditions Labor, 1986. 25-34.

Maeterlinck, Maurice. Les Aveugles. Fuvres. Marc Quaghebeur, préf. Bruxelles : Éditions Jacques Antoine, 1980. 33-82.

Maeterlinck, Maurice. «Le silence. » Le Trésor des humbles. Bruxelles : Éditions Labor, 1986. 13-23.

Maeterlinck, Maurice. « Menus Propos - Le théâtre. » Fuvres I. Paul Gorceix, éd. Bruxelles : Éditions Complexe, 1999. 457-463.

Maeterlinck, Maurice. «La vie profonde. » Le Trésor des humbles. Bruxelles : Éditions Labor, 1986. 135-148.

Novalis. Fragments. Maurice Maeterlinck, trad. Paul Gorceix, préf. Paris : Librairie José Corti, 1992.

Viegnes, Michel. «Le problème de l'inconnaissable dans les essais de Maeterlinck. » Annales Fondation Maurice Maeterlinck 18 (1991) : 67-81. 


\begin{abstract}
${ }^{1}$ Alors que plusieurs critiques se tournent vers la peinture fin de cerner les «débuts » de l'esthétique de l'abstraction, il est important de constater que certains penseurs des mouvements avant-gardistes considèrent que la littérature a fortement contribué au développement de l'abstraction dans l'art. Voici ce que considère André Breton, par exemple, dans «Genèse et perspectives du surréalisme » : « ce fut longtemps une grave lacune, à l'origine des mouvements - Dada, surréalisme - qui vont opérer la fusion de la poésie et des arts plastiques, de rendre complètement justice à Chagall. Les poètes eux-mêmes lui doivent beaucoup Apollinaire, à qui il a inspiré le poème peut-être le plus libre de ce siècle : 'À travers l'Europe', le Cendrars de la 'Prose du Transsibérien', il n'est pas jusqu'à Maïakovski et Essénine dont ne l'évoquent les plus hauts accents paroxystiques » (Breton 63). Kandinsky, à son tour, s'inspire de la poésie et des œuvres théâtrales de Maeterlinck en concevant Du Spirituel dans l'art.
\end{abstract}

${ }^{2}$ «En ce moment, le vent s'élève dans la forêt et la mer mugit, tout à coup et violemment, contre des falaises très voisines.

Premier aveugle-né : Il tonne !

Deuxième aveugle-né : Je crois que c'est une tempête qui s'élève.

La plus vieille aveugle : Je crois que c'est la mer.

Troisième aveugle-né : La mer ? - Est-ce que c'est la mer ? - Mais elle est à deux pas de nous ! - Elle est à côté

de nous ! Je l'entends tout autour de moi ! - Il faut que ce soit autre chose !

La jeune aveugle : J'entends le bruit des vagues à mes pieds.

Premier aveugle-né : Je crois que c'est le vent dans les feuilles mortes. » (Maeterlinck Aveugles 67) 